\title{
Fixed Points of Subadditive Maps with an Application to a System of Volterra-Fredholm Type Integrodifferential Equations
}

\author{
Hüseyin Işık (D), ${ }^{1,2}$ Bahman Moeini $\mathbb{D}^{3},{ }^{3}$ Hassen Aydi $\mathbb{D}^{4,},{ }^{4,5}$ and Nabil Mlaiki ${ }^{6}{ }^{6}$ \\ ${ }^{1}$ Nonlinear Analysis Research Group, Ton Duc Thang University, Ho Chi Minh City 700000, Vietnam \\ ${ }^{2}$ Faculty of Mathematics and Statistics, Ton Duc Thang University, Ho Chi Minh City 700000, Vietnam \\ ${ }^{3}$ Department of Mathematics, Hidaj Branch, Islamic Azad University, Hidaj, Iran \\ ${ }^{4}$ Université de Sousse, Institut Supérieur d'Informatique et des Techniques de Communication, Hammam Sousse 4000, Tunisia \\ ${ }^{5}$ China Medical University Hospital, China Medical University, Taichung 40402, Taiwan \\ ${ }^{6}$ Department of Mathematics and General Sciences, Prince Sultan University, Riyadh 11586, Saudi Arabia
}

Correspondence should be addressed to Hüseyin Işık; huseyin.isik@tdtu.edu.vn and Hassen Aydi; hassen.aydi@isima.rnu.tn

Received 11 June 2019; Revised 14 September 2019; Accepted 20 September 2019; Published 4 November 2019

Academic Editor: Leonid Shaikhet

Copyright (๑) 2019 Hüseyin Işık et al. This is an open access article distributed under the Creative Commons Attribution License, which permits unrestricted use, distribution, and reproduction in any medium, provided the original work is properly cited.

In this paper, some fixed-point theorems are established for strongly subadditive maps on $C(\Omega, \Upsilon)$ (where $C(\Omega, \Upsilon)$ denotes the space of $\Upsilon$-valued continuous functions on a compact Hausdorff space $\Omega$ and $\Upsilon$ is a unital Banach algebra). Finally, the result is applied to prove the existence and uniqueness of a solution for a system of nonlinear integrodifferential equations.

\section{Introduction}

Volterra-Fredholm integro differential equations [1-10] appear in a number of physical models, and an important question is whether these equations can support periodic solutions. This question has been studied extensively by a number of authors; in fact, they investigated the existence of solutions for a class of these kinds of equations using Schauder's fixed-point theorem and Green's function. The distance between two zeros of nontrivial solutions to integrodifferential equations was estimated by Domoshnitsky [11]. Ezzinbi and Ndambomve [12] considered the control system governed by some partial functional integrodifferential equations with finite delay in Banach spaces. On the other hand, the fixed-point theory is one of the most rapidly growing topics of nonlinear functional analysis. It is a vast and interdisciplinary subject whose study belongs to several mathematical domains such as classical analysis, functional analysis, operator theory, topology, and algebraic topology. This topic has grown very rapidly perhaps due to its interesting applications in various fields within and outside mathematics such as integral equations, initial and boundary value problems for ordinary and partial differential equations, and others. In our work, using a tool of fixed-point theory, we will ensure the existence and uniqueness of a solution for a system of nonlinear integrodifferential equations.

In [13], some properties of subadditive separating maps have been investigated. A fixed-point theorem was also given, and it was used to study the existence and uniqueness of a solution of nonlinear integral type equations. Consider $\Omega$ and $\Lambda$ as compact Hausdorff spaces. Take $\Upsilon$ as a unital Banach algebra. Denote by $C(\Omega, \Upsilon)($ resp. $C(\Lambda, \Upsilon))$ the set of $\Upsilon$-valued continuous functions on $\Omega$ (resp. $\Lambda$ ). The operator $\Delta: C(\Omega, \Upsilon) \longrightarrow C(\Lambda, \Upsilon)$ is said to be separating if $\|\zeta(\xi)\|$ $\cdot\|\varphi(\xi)\|=0$ implies that $\|\Delta \zeta(\varrho)\| \cdot\|\Delta \varphi(\varrho)\|=0$ for all $\zeta, \varphi \in C(\Omega, \Upsilon)$ and $\xi \in \Omega, \varrho \in \Lambda$. Recently, an attractive work on separating maps between different spaces of functions (as well as, operator algebras) is considered (see [14-17] and references therein). The existence, uniqueness, continuation, and other properties of solutions for nonlinear integral and integrodifferential equations are studied in [18-25].

The purpose of this work is to prove some new fixedpoint theorems for strongly subadditive maps and to ensure 
the existence and uniqueness of solutions for the following system of Volterra-Fredholm type integrodifferential equations:

$$
\left\{\begin{array}{l}
x^{\prime}(t)=\zeta(t)+\int_{0}^{t} k(t, s, x(s)) \mathrm{d} s+\int_{0}^{b} h(t, s, x(s)) \mathrm{d} s, \quad t \in J=[0, b], \\
x(0)=x_{0},
\end{array}\right.
$$

where $\zeta: J \longrightarrow \Omega, k, h: J \times J \times \Omega \longrightarrow \Omega$ are continuous and $x_{0} \in \Delta(\Delta$ is a Banach space with norm $\|\cdot\|)$. Note that a solution of problem (1) is as follows:

$$
\begin{aligned}
x(t)= & x_{0}+\int_{0}^{t} \zeta(s) \mathrm{d} s+\int_{0}^{t}\left[\int_{0}^{s} k(s, \varrho, x(\varrho)) \mathrm{d} \varrho\right. \\
& \left.+\int_{0}^{b} h(s, \varrho, x(\varrho)) \mathrm{d} \varrho\right] \mathrm{d} s, \quad t \in J=[0, b] .
\end{aligned}
$$

\section{Preliminaries}

In the sequel, denote by $B=C([0, b], \Omega)$ the Banach space of all continuous functions from $J$ into $\Omega$ endowed with the norm

$$
\|x\|=\sup _{s \in J}|x(s)|
$$

Let $\Omega$ and $\Lambda$ be two compact Hausdorff spaces. Let $\Upsilon$ be a unital Banach algebra with a unit element $e_{0}$. For $\zeta \in C(\Omega, \Upsilon)$, denote by $\operatorname{coz}(\zeta)$ the cozero set of $\zeta$, i.e., $\operatorname{coz}(\zeta)=\{\xi \in X: \zeta(\xi) \neq 0\}$. ClU (resp. CU) denotes the topological closure (resp. the compliment) of the set $U$. For a separating map $\Delta: C(\Omega, \Upsilon) \longrightarrow C(\Lambda, \Upsilon)$ and $\varrho \in \Lambda, \widehat{\varrho} \circ \Delta$ : $C(\Omega, \Upsilon) \longrightarrow \Upsilon$ and $\varrho \circ \Delta(f)=\Delta \zeta(\varrho)$, for all $\zeta \in C(\Omega, \Upsilon)$.

Definition 1 (see [26]).

(a) The operator $\Delta: C(\Omega, \Upsilon) \longrightarrow C(\Lambda, \Upsilon)$ is said to be subadditive, if

$$
\|\Delta(\zeta+\varphi)\| \leq\|\Delta \zeta\|+\|\Delta \varphi\|,
$$

for all $\zeta, \varphi \in C(\Omega, \Upsilon)$.

(b) Given $M>0$ and a subadditive map $\Delta$, if for any $\zeta \in C(\Omega, \Upsilon)$ there is $\varepsilon>0$ so that $\|\Delta \zeta-\delta \varphi\| \leq M\|\Delta(\zeta-\varphi)\|$, for all $\varphi \in C(\Omega, \Upsilon)$ verifying $\|\zeta-\varphi\|<\varepsilon$, then $\Delta$ is said to be strongly subadditive.

Example 1. Given a continuous map $\varphi: \Lambda \longrightarrow \Lambda$, take $\sigma \in C(\Lambda, \mathbb{C})$. The operator $\Delta: C(\Omega, \mathbb{C}) \longrightarrow C(\Lambda, \mathbb{C})$, defined by $\zeta \longrightarrow|\sigma \cdot(\zeta \circ \varphi)|$, is separating and strongly subadditive. Note that $\Delta$ it is not additive.

Definition 2 (see [26]). The operator $\Delta: C(\Omega, \Upsilon) \longrightarrow$ $C(\Lambda, \Upsilon)$ is said to be pointwise subadditive if

$$
\|\Delta(\zeta+\varphi)(\tau)\| \leq\|\Delta \zeta(\tau)\|+\|\Delta \varphi(\tau)\|,
$$

for all $\zeta, \varphi \in C(\Omega, \Upsilon)$ and $\tau \in \Lambda$.

Definition 3 (see [26]). Let $\Delta: C(\Omega, \Upsilon) \longrightarrow C(\Lambda, \Upsilon)$ be a separating map and $U$ be an open subset of $\Omega$. Such $U$ is said to be a vanishing set for $\widehat{\tau} \circ \delta$ if for each $\zeta \in C(\Omega, \Upsilon)$, $\operatorname{coz}(\zeta) \subseteq U$ implies that $\|\widehat{\tau} \Delta(\zeta)\|=0$. The support of $\widehat{\tau} \circ \Delta$ is given as follows:

$$
\operatorname{supp} \widehat{\tau} \circ \Delta=\frac{\Omega}{V \subseteq \Omega: V \text { is a vanishing set for } \widehat{\tau} \circ \Delta} .
$$

For $e \in \Upsilon$, define $1_{e}: \Omega \longrightarrow \Upsilon$ by $1_{e}(\xi)=e$ for each $\xi \in \Omega$. Here, $1_{e} \in C(\Omega, \Upsilon)$. Suppose that $\left\|\Delta 1_{e_{0}}(\tau)\right\| \neq 0$ for every $\tau \in \Lambda$. Due to the fact that $\left\|1_{e_{0}}\right\| \cdot 0=0$ and $\Delta$ is separating, we have $\Delta 0=0$. The use of $1_{e}$ is to extend the scalar-valued case to vector-valued case.

Remark 1 (decomposition of the identity). If $K=\mathbb{R}$ or $\mathbb{C}$, then for each finite cover $\left\{U_{i}\right\}_{i=1}^{n}$ of open subsets of $\Omega$, there exists a continuous decomposition of identity, $\left\{e_{i}\right\}_{i=1}^{n} \subseteq C(\Omega, \Upsilon)$, subordinate to the $U_{i}$. That is, $\sum_{i=1}^{n} e_{i}=1$ and $\operatorname{coz}\left(e_{i}\right) \subseteq U_{i}$, for any $i=1,2, \ldots, n$ [27].

Note that $\Delta 1_{e_{0}}$ is not equal to zero everywhere, so $\Delta(C(\Omega, \Upsilon))$ separates $\Lambda$. Also, each compact Hausdorff space is a Tychonoff space. The following remark is based on Theorem 1.1 of [28].

Remark 2. Let $\Delta: C(\Omega, \Upsilon) \longrightarrow C(\Lambda, \Upsilon)$ be a pointwise subadditive separating map. Then, for each $\tau \in \Lambda$, supp $(\widehat{\tau} \circ \Delta)$ is a singleton.

Theorem 1 (see [13]). Let $\Delta: C(\Omega, \Upsilon) \longrightarrow C(\Omega, \Upsilon)$ be a strongly subadditive map. Suppose that the following assumptions hold:

(A1) For any $\zeta \in C(\Omega, \Upsilon)$, there is $\varepsilon>0$ such that $\|\Delta \zeta-\zeta\|<\varepsilon$

(A2) There is $c>0$ such that $0<c M<1$ and $\|\Delta \zeta\| \leq c\|\zeta\|$, for any $\zeta \in C(\Omega, \Upsilon)$, where $M$ follows using the strong subadditivity of $\Delta$

Then, $\Delta$ possesses a unique fixed point $\zeta_{0}$ and $\lim _{n \rightarrow \infty} \Delta^{n} \zeta=\zeta_{0}$, for any $\zeta \in C(\Omega, \Upsilon)$.

Examples A.1. and A.2. given in Appendix support Theorem 1.

\section{Main Results}

Consider the following statement:

$(H)$ Let $y_{0}=\zeta$. Define in $C(\Omega, \Upsilon)$ a sequence $\left\{y_{n}\right\}$ approximately to $\Delta\left(y_{n-1}\right)$, i.e., $\left\{y_{n}\right\}$ satisfies

$$
\left\|y_{n}-\Delta\left(y_{n-1}\right)\right\| \leq \sigma_{n}
$$

where $\left\{\sigma_{n}\right\}$ is a sequence of positive numbers, for which $\lim _{n \longrightarrow \infty} \sigma_{n}=0$. 
Theorem 2. Let $\Delta: C(\Omega, \Upsilon) \longrightarrow C(\Omega, \Upsilon)$ be a strongly subadditive map. Suppose that the following assumptions hold:

(B1) For each $\zeta \in C(\Omega, \Upsilon)$, there is $\varepsilon>0$ such that $\left\|\Delta^{n}(\zeta)-y_{n-1}<\varepsilon\right\|$, for all $n \geq 1$, where $\left\{y_{n}\right\}$ is defined in $(H)$

(B2) There is $c>0$ such that $0<c M<1$ and $\|\Delta \zeta\| \leq c\|\zeta\|$, for each $\zeta \in C(\Omega, \Upsilon)$, where $M$ comes from strong subadditivity of $\Delta$

Then, $\Delta$ admits a unique fixed point $\zeta_{0}$ in $C(\Omega, \Upsilon)$. Moreover, for each $\zeta \in C(\Omega, \Upsilon), \lim _{n \longrightarrow \infty} \Delta^{n} \zeta=\zeta_{0}$.

Proof. It is sufficient to take $n=1$; then, by Theorem $1, \Delta$ has a unique fixed point $\zeta_{0}$ in $C(\Omega, \Upsilon)$. Also, for each $\zeta \in C(\Omega, \Upsilon), \lim _{n \longrightarrow \infty} \Delta^{n} \zeta=\zeta_{0}$.

Now, a natural question arises. If in Theorem 2, one replaces the sequence $\left\{\Delta^{n} \zeta\right\}$ by $\left\{y_{n}\right\}$, then under what conditions we have the case that

$$
\lim _{n \longrightarrow \infty} y_{n}=\zeta_{0} \text { ? }
$$

In the following, we propose a positive answer to this question.

Theorem 3. Consider the assumptions of Theorem 2 and let $\zeta_{0}$ be the unique fixed point of $\Delta$. Then, $\lim _{n \longrightarrow \infty} y_{n}=\zeta_{0}$, where $\left\{y_{n}\right\}$ is defined by $(H)$.

Proof. Let $y_{0}=\zeta$. By strong subadditivity of $\Delta$ and conditions (B1) and (B2), we have

$$
\begin{aligned}
\left\|\Delta^{m+1}\left(y_{0}\right)-y_{m}\right\|= & \left\|\Delta^{m+1}(\zeta)-y_{m}\right\| \\
\leq & \left\|\Delta\left(\Delta^{m}(\zeta)\right)-H\left(y_{m-1}\right)\right\| \\
& +\left\|\Delta\left(y_{m-1}\right)-y_{m}\right\| \\
\leq & M\left\|\Delta\left(\Delta^{m}(\zeta)-y_{m-1}\right)\right\|+\sigma_{m} \\
\leq & c M\left\|\Delta^{m}(\zeta)-y_{m-1}\right\|+\sigma_{m} \\
\leq & c M\left(c M\left\|\Delta^{m-1}(\zeta)-y_{m-2}\right\|+\sigma_{m-1}\right)+\sigma_{m} \\
& \vdots \\
\leq & c M\left(c M\left(\cdots\left(c M\left\|\Delta(\zeta)-y_{0}\right\|+\sigma_{1}\right) \cdots\right)\right. \\
& \left.+\sigma_{m-1}\right)+\sigma_{m},
\end{aligned}
$$

where $M, c>0, M$ comes from strong subadditivity of $\Delta$, and $0<c M<1$. We get that

$$
\left\|\Delta^{m+1}(\zeta)-y_{m}\right\| \leq \sum_{i=1}^{m}(c M)^{m-i} \sigma_{i} .
$$

Thus,

$$
\begin{aligned}
\left\|y_{m}-\zeta_{0}\right\| & \leq\left\|y_{m}-\Delta^{m+1}(\zeta)\right\|+\left\|\Delta^{m+1}(\zeta)-\zeta_{0}\right\| \\
& \leq \sum_{i=1}^{m}(c M)^{m-i} \sigma_{i}+\left\|\Delta^{m+1}(\zeta)-\zeta_{0}\right\| .
\end{aligned}
$$

Now, let $\sigma>0$. Since $\lim _{n \longrightarrow \infty} \sigma_{n}=0$, there is $N \in \mathbb{N}$ so that for $m \geq N, \sigma_{m} \leq \sigma$. Thus,

$$
\begin{aligned}
\sum_{i=1}^{m}(c M)^{m-i} \sigma_{i} & =\sum_{i=1}^{N}(c M)^{m-i} \sigma_{i}+\sum_{i=N+1}^{m}(c M)^{m-i} \sigma_{i} \\
& \leq(c M)^{m-N} \sum_{i=1}^{N}(c M)^{N-i} \sigma_{i}+\sigma \sum_{i=N+1}^{m}(c M)^{m-i} .
\end{aligned}
$$

Therefore,

$$
\lim _{m \longrightarrow \infty} \sum_{i=1}^{m}(c M)^{m-i} \sigma_{i} \leq \sigma\left(\frac{(c M)^{N+1}}{1-c M}\right) .
$$

Since $\sigma>0$ is arbitrary, $\lim _{m \rightarrow \infty} \sum_{i=1}^{m}(c M)^{m-i} \sigma_{i}=0$. Also, by Theorem 2, $\lim _{m \longrightarrow \infty}\left\|\Delta^{m+1}(\zeta)-\zeta_{0}\right\|=0$. Thus, $\lim _{m \rightarrow \infty} y_{m}=\zeta_{0}$.

Theorem 4. Suppose $\Delta: C(\Omega, \Upsilon) \longrightarrow C(\Omega, \Upsilon)$ is a map such that all the conditions of Theorem 1 are satisfied for $\Delta^{N}$ $\left(\Delta^{N}\right.$ is Nth iteration of $\Delta$ for some positive integer $\left.N\right)$. Then, $\Delta$ admits a unique fixed point.

Proof. By Theorem 1, $\Delta^{N}: C(\Omega, \Upsilon) \longrightarrow C(\Omega, \Upsilon)$ has a unique fixed point $\zeta \in C(\Omega, \Upsilon)$. However,

$$
\Delta^{N}(\Delta \zeta)=\Delta^{N+1} \zeta=\Delta\left(\Delta^{N} \zeta\right)=\Delta \zeta
$$

so $\Delta \zeta$ is also fixed point of $\Delta^{N}$. Since the fixed point of $\Delta^{N}$ is unique, one writes that $\Delta \zeta=\zeta$. Also, if $\Delta \varphi=\varphi$, then $\Delta^{N} \varphi=\varphi$. By uniqueness, we obtain that $\zeta=\varphi$.

Now, we present an extension of Theorem 1 .

Let $\mathcal{S}$ denote the class of functions $\alpha: \mathbb{R}^{+} \longrightarrow[0,1)$ such that $k \alpha\left(t_{n}\right) \longrightarrow 1 \Longrightarrow t_{n} \longrightarrow 0$ and $k \alpha(t) \leq 1$ for every $t \in \mathbb{R}^{+}$(where $k>0$ ).

Theorem 5. Let $\Delta: C(\Omega, \Upsilon) \longrightarrow C(\Omega, \Upsilon)$ be a strongly subadditive map. Suppose that the following assumptions hold:

(C1) For all $\zeta \in C(\Omega, \Upsilon)$, there is $\varepsilon>0$ so that $\left\|\Delta^{n+1}(\zeta)-\Delta^{n}(\zeta)<\varepsilon\right\|$, for each $n \in \mathbb{N}$

(C2) There is $\alpha \in \mathcal{S}$ so that $0<M \alpha(\|\zeta\|)<1$ and for each $\zeta \in C(\Omega, \Upsilon),\|\Delta \zeta\| \leq \alpha(\|\zeta\|)\|\zeta\|$, where $M$ comes from strong subadditivity of $\Delta$

Then, $\Delta$ admits a unique fixed point $\zeta_{0}$ and $\lim _{n \rightarrow \infty} \Delta^{n} \zeta=\zeta_{0}$, for each $\zeta \in C(\Omega, \Upsilon)$.

Proof. Fix $\zeta \in C(\Omega, \Upsilon)$ and let $\zeta_{n}=\Delta^{n} \zeta$ for $n=1,2,3, \ldots$ The proof is divided in two steps.

Step 1. We claim that $\lim _{n \longrightarrow \infty}\left\|\zeta_{n+1}-\zeta_{n}\right\|=0$. 
By conditions (C1) and (C2) and strong subadditivity of $\Delta$, the sequence $\left\{\left\|\zeta_{n+1}-\zeta_{n}\right\|\right\}$ is monotonously decreasing and is bounded below. Hence, $\lim _{n \longrightarrow \infty}\left\|\zeta_{n+1}-\zeta_{n}\right\|=r \geq 0$. Suppose that $r \neq 0$. Using the strong subadditivity of $H$ and condition (C2), we have

$$
\begin{aligned}
\frac{\left\|\zeta_{n+2}-\zeta_{n+1}\right\|}{\left\|\zeta_{n+1}-\zeta_{n}\right\|} & =\frac{\left\|\Delta^{n+2}(\zeta)-\Delta^{n+1}(\zeta)\right\|}{\left\|\Delta^{n+1}(\zeta)-\Delta^{n}(\zeta)\right\|} \\
& \leq \frac{M\left\|\Delta\left(\Delta^{n+1}(\zeta)-\Delta^{n}(\zeta)\right)\right\|}{\left\|\Delta^{n+1}(\zeta)-\Delta^{n}(\zeta)\right\|} \\
& \leq \frac{M \alpha\left(\left\|\Delta^{n+1}(\zeta)-\Delta^{n}(\zeta)\right\|\right)\left\|\Delta^{n+1}(\zeta)-\Delta^{n}(\zeta)\right\|}{\left\|\Delta^{n+1}(\zeta)-\Delta^{n}(\zeta)\right\|} \\
& =M \alpha\left(\left\|\Delta^{n+1}(\zeta)-\Delta^{n}(\zeta)\right\|\right) \\
& =M \alpha\left(\left\|\zeta_{n+1}-\zeta_{n}\right\|\right), \quad n=1,2,3, \ldots
\end{aligned}
$$

Taking $n \longrightarrow \infty$, we get $1 \leq \lim _{n \longrightarrow \infty} M \alpha\left(\left\|\zeta_{n+1}-\zeta_{n}\right\|\right)$ $<1$ (by (C2)). Since $\alpha \in \mathcal{S}$, this implies that $r=0$, that is, a contradiction, and so step 1 is completed.

Step 2. We shall prove that $\left\{\zeta_{n}\right\}$ is a Cauchy sequence. Suppose that $\limsup _{m, n \rightarrow \infty}\left\|\zeta_{m}-\zeta_{n}\right\|>0$. By triangle inequality, strong subadditivity of $\Delta$, and condition (C2), we have

$$
\begin{aligned}
\left\|\zeta_{m}-\zeta_{n}\right\| & \leq\left\|\zeta_{m}-\zeta_{m+1}\right\|+\left\|\zeta_{m+1}-\zeta_{n+1}\right\|+\left\|\zeta_{n+1}-\zeta_{n}\right\| \\
& \leq\left\|\zeta_{m}-\zeta_{m+1}\right\|+M \alpha\left(\left\|\zeta_{m}-\zeta_{n}\right\|\right)\left\|\zeta_{m}-\zeta_{n}\right\|+\left\|\zeta_{n+1}-\zeta_{n}\right\| \\
& \leq\left(1-M \alpha\left(\left\|\zeta_{m}-\zeta_{n}\right\|\right)\right)^{-1}\left[\left\|\zeta_{m}-\zeta_{m+1}\right\|+\left\|\zeta_{n+1}-\zeta_{n}\right\|\right] .
\end{aligned}
$$

Under the assumption that $\lim \sup _{m, n} \longrightarrow \infty\left\|\zeta_{m}-\zeta_{n}\right\|>0$, Step 1 now implies that

$$
\limsup _{m, n \longrightarrow \infty}\left(1-M \alpha\left(\left\|\zeta_{m}-\zeta_{n}\right\|\right)\right)^{-1}=+\infty .
$$

Therefore,

$$
\limsup _{m, n \longrightarrow \infty} M \alpha\left(\left\|\zeta_{m}-\zeta_{n}\right\|\right)=1 .
$$

However, since $\alpha \in \mathcal{S}$, this implies that $\lim \sup _{m, n \rightarrow \infty}\left\|\zeta_{m}-\zeta_{n}\right\|=0$, which is again a contradiction. Therefore, Step 2 is established.

Now, let $\zeta \in C(\Omega, \Upsilon)$. Since $\left\{\zeta_{n}\right\}=\left\{\Delta^{n} \zeta\right\}$ is a Cauchy sequence in $C(\Omega, \Upsilon)$, which is complete, we have $\lim _{n \longrightarrow \infty} \Delta^{n} \zeta=\zeta_{0} \in C(\Omega, \Upsilon)$. The continuity of $\Delta$ yields that $\Delta \zeta_{0}=\zeta_{0}$. Now, we shall show that $\zeta_{0}$ is the unique fixed point. Let $\varphi \in C(\Omega, \Upsilon)$ be another fixed point of $\Delta$. So, $\zeta_{0}=\lim _{n \longrightarrow \infty} \Delta^{n} \varphi=\varphi$, and this completes the proof.

\section{An Application to Nonlinear Integrodifferential Equations}

Consider the following assumptions:

(i) For all $x, y \in C([0, b], \Omega)$,

$$
\begin{aligned}
& \mid x_{0}+\int_{0}^{t} \zeta(s) \mathrm{d} s+\int_{0}^{t}\left[\int_{0}^{s} k(s, \varsigma,(x+y)(\varsigma)) \mathrm{d} \varsigma\right. \\
& \left.\quad+\int_{0}^{b} h(s, \varsigma,(x+y)(\varsigma)) \mathrm{d} \varsigma\right] \mathrm{d} s \mid \\
& \leq \mid x_{0}+\int_{0}^{t} \zeta(s) \mathrm{d} s+\int_{0}^{t}\left[\int_{0}^{s} k(s, \varsigma, x(\varsigma)) \mathrm{d} \varsigma\right. \\
& \left.\quad+\int_{0}^{b} h(s, \varsigma, x(\varsigma)) \mathrm{d} \varsigma\right] \mathrm{d} s \mid \\
& \quad+\mid x_{0}+\int_{0}^{t} \zeta(s) \mathrm{d} s+\int_{0}^{t}\left[\int_{0}^{s} k(s, \varsigma, y(\varsigma)) \mathrm{d} \varsigma\right. \\
& \left.\quad+\int_{0}^{b} h(s, \varsigma, y(\varsigma)) \mathrm{d} \varsigma\right] \mathrm{d} s \mid
\end{aligned}
$$

for all $t \in[0, b]$.

(ii) Let $M>0$. For each $x \in C([0, b], \Omega)$, there is $\varepsilon>0$ such that

$$
\begin{aligned}
& \mid \int_{0}^{t}\left[\int_{0}^{s}(k(s, \varsigma, x(\varsigma))-k(s, \varsigma, y(\varsigma))) \mathrm{d} \varsigma\right. \\
& \left.\quad+\int_{0}^{b}(h(s, \varsigma, x(\varsigma))-h(s, \varsigma, y(\varsigma))) \mathrm{d} \varsigma\right] \mathrm{d} s \mid \\
& \leq M \mid x_{0}+\int_{0}^{t} \zeta(s) \mathrm{d} s+\int_{0}^{t}\left[\int_{0}^{s} k(s, \varsigma,(x-y)(\varsigma)) \mathrm{d} \varsigma\right. \\
& \left.\quad+\int_{0}^{b} h(s, \varsigma,(x-y)(\varsigma)) \mathrm{d} \varsigma\right] \mathrm{d} s \mid
\end{aligned}
$$

for each $y \in C([0, b], \Omega)$ satisfying $\|x-y\|<\varepsilon$ and for each $t \in[0, b]$.

(iii) For each $x \in C([0, b], \Omega)$, there is $\varepsilon>0$ such that

$$
\begin{gathered}
\| x_{0}+\int_{0}^{t} \zeta(s) \mathrm{d} s+\int_{0}^{t}\left[\int_{0}^{s} k(s, \varsigma, x(\varsigma)) \mathrm{d} \varsigma\right. \\
\left.+\int_{0}^{b} h(s, \varsigma, x(\varsigma)) \mathrm{d} \varsigma\right] \mathrm{d} s-x(t) \|<\varepsilon .
\end{gathered}
$$

(iv) For each $x \in C([0, b], \Omega)$, there is $L>0$ such that 


$$
\begin{gathered}
\| x_{0}+\int_{0}^{t} \zeta(s) \mathrm{d} s+\int_{0}^{t}\left[\int_{0}^{s} k(s, \varsigma, x(\varsigma)) \mathrm{d} \varsigma\right. \\
\left.+\int_{0}^{b} h(s, \varsigma, x(\varsigma)) \mathrm{d} \varsigma\right] \mathrm{d} s\|\leq L\| x \| .
\end{gathered}
$$

Theorem 6. Let $\Omega$ be a Banach space. Suppose that the assumptions (i) - (iv) hold. Then, the initial value problem (1) has a unique solution in $C([0, b], \Omega)$, for which $0<L M<1$.

Proof. We shall prove that the operator $G: C([0, b], \Omega) \longrightarrow C([0, b], \Omega)$, given as

$$
\begin{aligned}
G x(t)= & x_{0}+\int_{0}^{t} \zeta(s) \mathrm{d} s+\int_{0}^{t}\left[\int_{0}^{s} k(s, \varsigma, x(\varsigma)) \mathrm{d} \varsigma\right. \\
& \left.+\int_{0}^{b} h(s, \varsigma, x(\varsigma)) \mathrm{d} \varsigma\right] \mathrm{d} s, \quad t \in[0, b],
\end{aligned}
$$

admits a unique fixed point, which is a solution of (1). For this, by condition (i), we have

$$
\begin{aligned}
\|G(x+y)\|= & \| x_{0}+\int_{0}^{t} \zeta(s) \mathrm{d} s+\int_{0}^{t}\left[\int_{0}^{s} k(s, \varsigma,(x+y)(\varsigma)) \mathrm{d} \varsigma\right. \\
& \left.+\int_{0}^{b} h(s, \varsigma,(x+y)(\varsigma)) \mathrm{d} \varsigma\right] \mathrm{d} s \| \\
= & \sup _{0 \leq t \leq b} \mid x_{0}+\int_{0}^{t} \zeta(s) \mathrm{d} s+\int_{0}^{t}\left[\int_{0}^{s} k(s, \varsigma,(x+y)(\varsigma)) \mathrm{d} \varsigma\right. \\
& \left.+\int_{0}^{b} h(s, \varsigma,(x+y)(\varsigma)) \mathrm{d} \varsigma\right] \mathrm{d} s \mid \\
\leq & \sup _{0 \leq t \leq b} \mid x_{0}+\int_{0}^{t} \zeta(s) \mathrm{d} s+\int_{0}^{t}\left[\int_{0}^{s} k(s, \varsigma, x(\varsigma)) \mathrm{d} \varsigma\right. \\
& \left.+\int_{0}^{b} h(s, \varsigma, x(\varsigma)) \mathrm{d} \varsigma\right] \mathrm{d} s\left|+\sup _{0 \leq t \leq b}\right| x_{0} \\
& +\int_{0}^{t} \zeta(s) \mathrm{d} s+\int_{0}^{t}\left[\int_{0}^{s} k(s, \varsigma, y(\varsigma)) \mathrm{d} \varsigma\right. \\
& \left.+\int_{0}^{b} h(s, \varsigma, y(\varsigma)) \mathrm{d} \varsigma\right] \mathrm{d} s \mid \\
= & \|G x\|+\|G y\|,
\end{aligned}
$$

for all $x, y \in C([0, b], \Omega)$. Hence, $G$ is subadditive. Let $M>0$. By condition (ii), for each $x \in C([0, b], \Omega)$, there is $\epsilon>0$ such that

$$
\begin{aligned}
\|G x-G y\|= & \| \int_{0}^{t}\left[\int_{0}^{s} k(s, \varsigma, x(\varsigma)) \mathrm{d} \varsigma+\int_{0}^{b} h(s, \varsigma, x(\varsigma)) \mathrm{d} \varsigma\right] \mathrm{d} s \\
& -\int_{0}^{t}\left[\int_{0}^{s} k(s, \varsigma, y(\varsigma)) \mathrm{d} \varsigma+\int_{0}^{b} h(s, \varsigma, y(\varsigma)) \mathrm{d} \varsigma\right] \mathrm{d} s \| \\
= & \sup _{0 \leq t \leq b} \mid \int_{0}^{t}\left[\int_{0}^{s} k(s, \varsigma, x(\varsigma)) \mathrm{d} \varsigma+\int_{0}^{b} h(s, \varsigma, x(\varsigma)) \mathrm{d} \varsigma\right] \mathrm{d} s \\
& -\int_{0}^{t}\left[\int_{0}^{s} k(s, \varsigma, y(\varsigma)) \mathrm{d} \varsigma+\int_{0}^{b} h(s, \varsigma, y(\varsigma)) \mathrm{d} \varsigma\right] \mathrm{d} s \mid \\
= & \sup _{0 \leq t \leq b} \mid \int_{0}^{t}\left[\int_{0}^{s}(k(s, \varsigma, x(\varsigma))-k(s, \varsigma, y(\varsigma))) \mathrm{d} \varsigma\right. \\
& \left.+\int_{0}^{b}(h(s, \varsigma, x(\varsigma))-h(s, \varsigma, y(\varsigma))) \mathrm{d} \varsigma\right] \mathrm{d} s \mid \\
\leq & M \sup _{0 \leq t \leq b} \mid x_{0}+\int_{0}^{t} \zeta(s) \mathrm{d} s+\int_{0}^{t}\left[\int_{0}^{s} k(s, \varsigma,(x-y)(\varsigma)) \mathrm{d} \varsigma\right. \\
& \left.+\int_{0}^{b} h(s, \varsigma,(x-y)(\varsigma)) \mathrm{d} \varsigma\right] \mathrm{d} s \mid \\
= & M\|G(x-y)\|,
\end{aligned}
$$

for each $y \in C([0, b], \Omega)$ satisfying $\|x-y\|<\varepsilon$, so $G$ is strongly subadditive. By condition (iii), for each $x \in C([0, b], \Omega)$, there is $\varepsilon>0$ so that $\|G x-x\|<\varepsilon$. Also, by condition (iv), we conclude that for each $x \in C([0, b], \Omega)$, there is $L>0$ such that $\|G x\| \leq L\|x\|$, for which $0<L M<1$. Therefore, all conditions of Theorem 1 hold, and so (1) has a unique solution.

Now, an example is provided in order to make use of our result.

Example 2. Let $l_{a}(a \geq 2)$ be the map as in Example A.1. (given later in Appendix section). Take $\Omega=\mathbb{R}^{+} \cup\{0\}$ and $x \in C([0,1], \Omega)$. We consider the following initial value problem:

$$
\left\{\begin{aligned}
x^{\prime}(t)= & \frac{1}{\sqrt{1+\left(1200(5)^{2} / 1199\right)}}\left(\int_{0}^{t} t l_{s+2}(x(s)) \mathrm{d} s\right. \\
& \left.+\int_{0}^{1} s|x(s)| \mathrm{d} s\right), \quad t \in[0,1], \\
x(0)= & 0 .
\end{aligned}\right.
$$

For all $s, t \in[0,1]$ and $x \in C([0,1], \Omega)$, we define in (1): 


$$
\begin{gathered}
\zeta(t)=0, k(t, s, x(s))=\frac{1}{\sqrt{1+\left(1200(5)^{2} / 1199\right)}} t l_{s+2}(x(s)) \\
\text { and } h(t, s, x(s))=\frac{1}{\sqrt{1+\left(1200(5)^{2} / 1199\right)}} s|x(s)|,
\end{gathered}
$$$$
\text { and the norm }\|x-y\|=\sup _{0 \leq t \leq 1}|x(t)-y(t)| \quad \text { on }
$$$$
C([0,1], \Omega) \text {. Then, } C([0,1], \Omega) \text { is a Banach space. }
$$

(i) For all $x, y \in C([0,1], \Delta)$ and $t \in[0,1]$, we have

$$
\begin{aligned}
& \left|x_{0}+\int_{0}^{t} \zeta(s) \mathrm{d} s+\int_{0}^{t}\left[\int_{0}^{s} k(s, \varsigma,(x+y)(\varsigma)) \mathrm{d} \varsigma+\int_{0}^{1} h(s, \varsigma,(x+y)(\varsigma)) \mathrm{d} \varsigma\right] \mathrm{d} s\right| \\
& =\frac{1}{\sqrt{1+\left(1200(5)^{2} / 1199\right)}}\left|\int_{0}^{t}\left[\int_{0}^{s} s l_{\varsigma+2}((x+y)(\varsigma)) \mathrm{d} \varsigma+\int_{0}^{1} \varsigma|(x+y)(\varsigma)| \mathrm{d} \varsigma\right] \mathrm{d} s\right| \\
& \leq \frac{1}{\sqrt{1+\left(1200(5)^{2} / 1199\right)}}\left|\int_{0}^{t}\left[\int_{0}^{s} s l_{\varsigma+2}(x(\varsigma)) \mathrm{d} \varsigma+\int_{0}^{1} \varsigma|x(\varsigma)| \mathrm{d} \varsigma\right] \mathrm{d} s\right| \\
& \quad+\frac{1}{\sqrt{1+\left(1200(5)^{2} / 1199\right)}}\left|\int_{0}^{t}\left[\int_{0}^{s} s l_{\zeta+2}(y(\varsigma)) \mathrm{d} \varsigma+\int_{0}^{1} \varsigma|y(\varsigma)| \mathrm{d} \varsigma\right] \mathrm{d} s\right| \\
& =\left|x_{0}+\int_{0}^{t} \zeta(s) \mathrm{d} s+\int_{0}^{t}\left[\int_{0}^{s} k(s, \varsigma, x(\varsigma)) \mathrm{d} \varsigma+\int_{0}^{1} h(s, \varsigma, x(\varsigma)) \mathrm{d} \varsigma\right] \mathrm{d} s\right| \\
& \quad+\left|x_{0}+\int_{0}^{t} \zeta(s) \mathrm{d} s+\int_{0}^{t}\left[\int_{0}^{s} k(s, \varsigma, x(\varsigma)) \mathrm{d} \varsigma+\int_{0}^{1} h(s, \varsigma, x(\varsigma)) \mathrm{d} \varsigma\right] \mathrm{d} s\right|
\end{aligned}
$$

(ii) For every $t \in[0,1]$, we have

$$
\begin{aligned}
& \left|\int_{0}^{t}\left[\int_{0}^{s}(k(s, \varsigma, x(\varsigma))-k(s, \varsigma, y(\varsigma))) \mathrm{d} \varsigma+\int_{0}^{1}(h(s, \varsigma, x(\varsigma))-h(s, \varsigma, y(\varsigma))) \mathrm{d} \varsigma\right] \mathrm{d} s\right| \\
& =\frac{1}{\sqrt{1+\left(1200(5)^{2} / 1199\right)}}\left|\int_{0}^{t}\left[\int_{0}^{s}\left(s l_{\varsigma+2}(x(\varsigma))-s l_{\varsigma+2}(y(\varsigma))\right) \mathrm{d} \varsigma+\int_{0}^{1}(\varsigma|x(\varsigma)|-\varsigma|y(\varsigma)|) \mathrm{d} \varsigma\right] \mathrm{d} s\right| \\
& \leq \frac{1}{\sqrt{1+\left(1200(5)^{2} / 1199\right)}}\left|\int_{0}^{t}\left[\int_{0}^{s} \sqrt{1+\frac{1200(1+\varsigma)^{2}}{1199}} s l_{\zeta+2}((x-y)(\varsigma)) \mathrm{d} \varsigma+\int_{0}^{1} \varsigma|x(\varsigma)-y(\varsigma)| \mathrm{d} \varsigma\right] \mathrm{d} s\right| \\
& \leq \frac{\sqrt{1+\left(1200(3)^{2} / 1199\right)}}{\sqrt{1+\left(1200(5)^{2} / 1199\right)}}\left|\int_{0}^{t}\left[\int_{0}^{s} s l_{\varsigma+2}((x-y)(\varsigma)) \mathrm{d} \varsigma+\int_{0}^{1} \varsigma|x(\varsigma)-y(\varsigma)| \mathrm{d} \varsigma\right] \mathrm{d} s\right| \\
& =M\left|x_{0}+\int_{0}^{t} \zeta(s) \mathrm{d} s+\int_{0}^{t}\left[\int_{0}^{0} k(s, \varsigma,(x-y)(\varsigma)) \mathrm{d} \varsigma+\int_{0}^{1} h(s, \varsigma,(x-y)(\varsigma)) \mathrm{d} \varsigma\right] \mathrm{d} s\right|
\end{aligned}
$$

with $M=\sqrt{1+\left(1200(3)^{2} / 1199\right)}$ and for each $y \in C([0,1], \Omega)$ satisfying $\|x-y\|<\varepsilon$.

(iii) For every $x \in C([0,1], \Omega)$, we have 


$$
\begin{aligned}
& \left\|x_{0}+\int_{0}^{t} \zeta(s) \mathrm{d} s+\int_{0}^{t}\left[\int_{0}^{s} k(s, \varsigma, x(\varsigma)) \mathrm{d} \varsigma+\int_{0}^{1} h(s, \varsigma, x(\varsigma)) \mathrm{d} \varsigma\right] \mathrm{d} s-x(t)\right\| \\
& =\left\|\frac{1}{\sqrt{1+\left(1200(5)^{2} / 1199\right)}} \int_{0}^{t}\left[\int_{0}^{s} s l_{\varsigma+2}(x(\varsigma)) \mathrm{d} \varsigma+\int_{0}^{1} \varsigma|x(\varsigma)| \mathrm{d} \varsigma\right] \mathrm{d} s-x(t)\right\| \\
& \leq\left\|\frac{1}{\sqrt{1+\left(1200(5)^{2} / 1199\right)}} \int_{0}^{t}\left[\int_{0}^{s} s l_{\varsigma+2}(x(\varsigma)) \mathrm{d} \varsigma+\int_{0}^{1} \varsigma|x(\varsigma)| \mathrm{d} \varsigma\right] \mathrm{d} s\right\|+\|x(t)\| \\
& \leq \sup _{0 \leq t \leq 1}\left|\int_{0}^{t}\left[\int_{0}^{s} s|x(\varsigma)| \mathrm{d} \varsigma+\int_{0}^{1} \varsigma|x(\varsigma)| \mathrm{d} \varsigma\right] \mathrm{d} s\right|+M_{1} \\
& \leq \sup _{0 \leq t \leq 1}\left|\int_{0}^{t}\left[s^{2} M_{1}+\frac{1}{2} M_{1}\right] \mathrm{d} s\right|+M_{1} \\
& <2 M_{1}=\epsilon .
\end{aligned}
$$

Again by (iii), we have

$$
\begin{aligned}
& \| x_{0}+\int_{0}^{t} \zeta(s) \mathrm{d} s+\int_{0}^{t}\left[\int_{0}^{s} k(s, \varsigma, x(\varsigma)) \mathrm{d} \varsigma\right. \\
& \left.\quad+\int_{0}^{1} h(s, \varsigma, x(\varsigma)) \mathrm{d} \varsigma\right] \mathrm{d} s \| \\
& \leq \frac{1}{\sqrt{1+\left(1200(5)^{2} / 1199\right)}}\left(\frac{5}{6}\|x\|\right) \\
& <\frac{1}{\sqrt{1+\left(1200(5)^{2} / 1199\right)}}\|x\| .
\end{aligned}
$$

Therefore,

$$
\begin{gathered}
\| x_{0}+\int_{0}^{t} \zeta(s) \mathrm{d} s+\int_{0}^{t}\left[\int_{0}^{s} k(s, \varsigma, x(\varsigma)) \mathrm{d} \varsigma\right. \\
\left.+\int_{0}^{1} h(s, \varsigma, x(\varsigma)) \mathrm{d} \varsigma\right] \mathrm{d} s\|\leq L\| x \|,
\end{gathered}
$$

with $L=1 /\left(\sqrt{1+\left(1200(5)^{2} / 1199\right)}\right)$. Also, $\quad 0<L M=$ $\left(\sqrt{1+\left(1200(3)^{2} / 1199\right)}\right) /\left(\sqrt{1+\left(1200(5)^{2} / 1199\right)}\right)<1$. Thus, all conditions of Theorem 6 hold, and so the initial value problem (26) possesses a unique solution in $C([0,1], \Omega)$.

\section{Conclusion}

We established some fixed-point theorems for strongly subadditive maps. We applied the obtained results to ensure the existence and uniqueness of a solution for a system of nonlinear integrodifferential equations. Moreover, some related examples have been presented in support of the given results.

\section{Appendix}

The following examples are in support of Theorem 1 .

Example A.1. Given $a \geq 2$ and $z=x e^{i \theta} \in \mathbb{C}$ with $-\pi<\theta<\pi$, take $l_{a}: \mathbb{C} \longrightarrow \mathbb{C}$ given as

$$
l_{a}: x e^{i \theta} \longmapsto \frac{1}{2+(2 \sqrt{2} a)^{2}} x e^{i a \theta} .
$$

Then, $l_{a}$ is subadditive and strongly subadditive. Here, 0 is the unique fixed point of $l_{a}$.

For this, since $\left|l_{a}(z)\right|=\left(1 /\left(2+(2 \sqrt{2} a)^{2}\right)\right)|z|$, it follows that

$$
\begin{aligned}
\left|l_{a}\left(z+z^{\prime}\right)\right| & =\frac{1}{2+(2 \sqrt{2} a)^{2}}\left|z+z^{\prime}\right| \\
& \leq \frac{1}{2+(2 \sqrt{2} a)^{2}}|z|+\frac{1}{2+(2 \sqrt{2} a)^{2}}\left|z^{\prime}\right| \\
& =\left|l_{a}(z)\right|+\left|l_{a}\left(z^{\prime}\right)\right| .
\end{aligned}
$$

Therefore, $l_{a}$ is subadditive. The continuity of $l_{a}$ is clear. To show that $l_{a}$ is strongly subadditive: given any $z_{0} \in \mathbb{C}$, we should find $\epsilon_{z_{0}}>0$ and $M>0$ so that $\left|l_{a}(z)-l_{a}\left(z_{0}\right)\right| \leq M\left|l_{a}\left(z-z_{0}\right)\right|$ when $\left|z-z_{0}\right|<\varepsilon_{z_{0}}$. It suffices to ensure that

$$
f\left(z, z_{0}\right)=\frac{\left|l_{a}(z)-l_{a}\left(z_{0}\right)\right|^{2}}{\left(2+(2 \sqrt{2} a)^{2}\right)^{2}\left|z-z_{0}\right|^{2}}
$$


is bounded in some neighborhood of $z_{0}$. Let $z=x e^{i \theta}$ and $z_{0}=x_{0} e^{i \theta_{0}}$. Note that

$$
\begin{aligned}
f\left(z, z_{0}\right) & =\frac{\left|\left(1 /\left(2+(2 \sqrt{2} a)^{2}\right)\right) x e^{i a \theta}-\left(1 /\left(2+(2 \sqrt{2} a)^{2}\right)\right) x_{0} e^{i a \theta_{0}}\right|^{2}}{\left(2+(2 \sqrt{2} a)^{2}\right)^{2}\left|x e^{i \theta}-x_{0} e^{i \theta_{0}}\right|^{2}} \\
& =\frac{\left|\left(x e^{i a \theta} / x_{0} e^{i a \theta_{0}}\right)-1\right|^{2}}{\left|\left(x e^{i \theta} / x_{0} e^{i \theta_{0}}\right)-1\right|^{2}}=f\left(\frac{z}{z_{0}}, 1\right) .
\end{aligned}
$$

First, we consider the case $z_{0}=1$. Then,

$$
f(z, 1)=\frac{\left|1-x e^{i a \theta}\right|^{2}}{\left|1-x e^{i \theta}\right|^{2}}=\frac{1+x^{2}-2 x \cos (a \theta)}{1+x^{2}-2 x \cos (\theta)} .
$$

Now, we show that $f(z, 1)$ is bounded from above for $z$ in a neighborhood of 1 . Using power series expansion of $\cos (\theta)$, we get

$$
\begin{aligned}
& 1-\frac{\theta^{2}}{2}<\cos (\theta)<1-\frac{\theta^{2}}{2}+\frac{\theta^{4}}{24}, \\
& \frac{\theta^{2}}{2}-1>-\cos (\theta)>-1+\frac{\theta^{2}}{2}-\frac{\theta^{4}}{24} .
\end{aligned}
$$

These inequalities imply that

$$
\begin{aligned}
f(z, 1) & <\frac{1+x^{2}+2 x\left(\left(a^{2} \theta^{2} / 2\right)-1\right)}{1+x^{2}+2 x\left(\left(\theta^{2} / 2\right)-1-\theta^{4} / 24\right)} \\
& =\frac{(1-x)^{2}+x a^{2} \theta^{2}}{(1-x)^{2}+2 x\left(\left(\theta^{2} / 2-\left(\theta^{4} / 24\right)\right)\right)} .
\end{aligned}
$$

For $|\theta|<(1 / 10)$, we have $\left(\theta^{2} / 2\right)-\left(\theta^{4} / 24\right)>0$ and

$$
\begin{aligned}
f(z, 1) & <\frac{(1-x)^{2}+x a^{2} \theta^{2}}{(1-x)^{2}+2 x\left(\left(\theta^{2} / 2\right)-\left(\theta^{4} / 24\right)\right)} \\
& <1+\frac{a^{2}}{2\left((1 / 2)-\left(\theta^{2} / 24\right)\right)} .
\end{aligned}
$$

If $|\theta|<(1 / 10)$, then $(1 / 2)-\left(\theta^{2} / 24\right)>(1199 / 2400)$, and it follows that $f(z, 1)<1+\left(1200 a^{2} / 1199\right)$. Thus, $\sqrt{f(z, 1)}<\sqrt{1+\left(1200 a^{2} / 1199\right)}$. Now, suppose that $z_{0} \neq 0$ or 1. Choose $\varepsilon>0$ so that if $\left|\left(z / z_{0}\right)-1\right|<\varepsilon$, then $\arg \left(z / z_{0}\right)<(1 / 10)$. Since $f\left(z, z_{0}\right)=f\left(z / z_{0}, 1\right)$, we get $\sqrt{f\left(z, z_{0}\right)}<\sqrt{1+\left(1200 a^{2} / 1199\right)}$ for $\left|z-z_{0}\right|<\varepsilon\left|z_{0}\right|$. After including the case $z_{0}=0$, we have

$$
M=\max \left\{1, \sqrt{1+\frac{1200 a^{2}}{1199}}\right\}=\sqrt{1+\frac{1200 a^{2}}{1199}} .
$$

Also,

(A1) For any $z \in \mathbb{C}$, there is $\varepsilon>0$ so that $\left|l_{a}(z)-z\right|<\varepsilon$

(A2) For any $z=x e^{i \theta} \in C$, we have

$$
\left|l_{a}(z)\right|=\frac{1}{2+(2 \sqrt{2} a)^{2}} x<\frac{1}{2+2 \sqrt{2} a^{2}} x=\frac{1}{2+2 \sqrt{2} a^{2}}|z| .
$$

Choosing $\quad c=1 /\left(2+2 \sqrt{2} a^{2}\right), \quad 0<c M=(1 /(2+$ $\left.\left.2 \sqrt{2} a^{2}\right)\right) \sqrt{1+\left(1200 a^{2} / 1199\right)}<1$ and $\left|l_{a}(z)\right|<c|z|$. Thus, all conditions of Theorem 1 hold and $z=0$ is the unique fixed point of $l_{a}$. Moreover, for each $z \in \mathbb{C}, \lim _{n \longrightarrow \infty} l_{a}^{n}(z)=0$.

Example A.2. Let $l_{a}(a \geq 2)$ be as in Example A.1. and $\Delta$ : $C([0,1], \mathbb{C}) \longrightarrow C([0,1], \mathbb{C})$ be given as

$$
\Delta x(s)=l_{s+2}(x(s)), \quad s \in[0,1] .
$$

Clearly, $\Delta$ is a subadditive and strongly subadditive map.

\section{Data Availability}

The data used to support the findings of this study are available from the corresponding author upon request.

\section{Conflicts of Interest}

The authors declare that they have no conflicts of interest.

\section{Authors' Contributions}

All authors contributed equally and significantly in writing this article. All authors read and approved the final manuscript.

\section{Acknowledgments}

Nabil Mlaiki would like to thank Prince Sultan University for funding this work through the research group "Nonlinear Analysis Methods in Applied Mathematics" (NAMAM) (group no. RG-DES-2017-01-17).

\section{References}

[1] K. Maleknejad and M. Hadizadeh, "A new computational method for Volterra-Fredholm integral equations," Computers \& Mathematics with Applications, vol. 37, no. 9, pp. 1-8, 1999.

[2] K. Maleknejad and Y. Mahmoudi, "Taylor polynomial solution of high-order nonlinear Volterra-Fredholm integrodifferential equations," Applied Mathematics and Computation, vol. 145, no. 2-3, pp. 641-653, 2003.

[3] K. Nouri, M. Nazari, B. Keramati, and L. Torkzadeh, "Existence and uniqueness of solutions for a class of functionalintegral equations with fractional derivative and time-dependent delay," Journal of Nonlinear Functional Analysis, vol. 2018, pp. 1-13, 2018.

[4] S. A. Yousefi, A. Lotfi, and M. Dehghan, "He's variational iteration method for solving nonlinear mixed VolterraFredholm integral equations," Computers \& Mathematics with Applications, vol. 58, no. 11-12, pp. 2172-2176, 2009.

[5] A.-M. Wazwaz, "A reliable treatment for mixed VolterraFredholm integral equations," Applied Mathematics and Computation, vol. 127, no. 2-3, pp. 405-414, 2002. 
[6] H. Aydi, H. R. Marasi, H. Piri, and A. Talebi, "A solution to the new caputo-fabrizio fractional KDV equation via stability," Journal of Mathematical Analysis, vol. 8, no. 4, pp. 147-155, 2017.

[7] H. Aydi, T. Wongyat, and W. Sintunavarat, "On new evolution of Ri's result via $w$-distances and the study on the solution for nonlinear integral equations and fractional differential equations," Advances in Differences Equations, vol. 2018, no. 1, p. 132, 2018.

[8] D. Baleanu, S. Rezapour, and H. Mohammadi, "Some existence results on nonlinear fractional differential equations," Philosophical Transactions of the Royal Society A: Mathematical, Physical and Engineering Sciences, vol. 371, no. 1990, Article ID 20120144, 2013.

[9] H. Marasi, H. Piri, and H. Aydi, "Existence and multiplicity of solutions for nonlinear fractional differential equations," Journal of Nonlinear Sciences and Applications, vol. 9, no. 6, pp. 4639-4646, 2016.

[10] B. Samet and H. Aydi, "On some inequalities involving liouville-caputo fractional derivatives and applications to special means of real numbers," Mathematics, vol. 6, no. 10, p. 193, 2018.

[11] A. Domoshnitsky, "Sturm theorems and distance between adjacent zeros for second order integro-differential equations," Journal of Nonlinear and Variational Analysis, vol. 2, no. 2, pp. 155-164, 2018.

[12] K. Ezzinbi and P. Ndambomve, "On the solvability and optimal control of some partial functional integrodifferential equations with infinite delay in Banach spaces," Communications in Optimization Theory, vol. 2018, pp. 1-18, 2018.

[13] Y. Estaremi and B. Moeini, "Fixed points of subadditive maps and some non-linear integral equations," Journal of Fixed Point Theory and Applications, vol. 19, no. 2, pp. 1269-1282, 2017.

[14] J. Araujo and K. Jarosz, "Automatic continuity of biseparating maps,” Studia Mathematica, vol. 155, no. 3, pp. 231-239, 2003.

[15] J. J. Font and S. Hernandez, "On separating maps between locally compact spaces," Archiv der Mathematik, vol. 63, no. 2, pp. 158-165, 1994.

[16] K. Jarosz, "Automatic continuity of separating linear isomorphisms," Canadian Mathematical Bulletin, vol. 33, no. 2, pp. 139-144, 1990.

[17] F. Sady and Y. Estaremi, "Subadditive separating maps between regular Banach function algebras," Bulletin of the Korean Mathematical Society, vol. 44, no. 4, pp. 753-761, 2007.

[18] R. P. Agarwal, N. Hussain, and M. A. Taoudi, "Fixed point theorems in ordered Banach spaces and applications to nonlinear integral equations," Abstract and Applied Analysis, vol. 2012, Article ID 245872, 15 pages, 2012.

[19] A. Karoui, "On the existence of continuous solutions of nonlinear integral equations," Applied Mathematics Letters, vol. 18, no. 3, pp. 299-305, 2005.

[20] B. Moeini and A. Razani, "JH-operator pairs of type $(R)$ with application to nonlinear integral equations," Vietnam Journal of Mathematics, vol. 43, no. 4, pp. 777-792, 2015.

[21] B. Moeini, A. H. Ansari, and C. Park, "JHR-operator pairs in $\mathrm{C}^{*}$-algebra-valued modular metric spaces and related fixed point results via $C^{*}$-class functions," Journal of Fixed Point Theory and Applications, vol. 20, no. 1, 2018.

[22] B. Moeini, A. H. Ansari, and C. Park, "JHR-operator pairs in $\mathrm{C}^{*}$-algebra-valued modular metric spaces and related fixed point results with application," Numerical Functional Analysis and Optimization, vol. 39, no. 16, pp. 1785-1805, 2018.
[23] B. Moeini, A. H. Ansari, and H. Aydi, "Some common fixed point theorems without orbital continuity via $C$-class functions and an application," Journal of Mathematical Analysis, vol. 8 , no. 4, pp. 46-55, 2017.

[24] B. G. Pachpatte, "On Fredholm type integrodifferential equation," Tamkang Journal of Mathematics, vol. 39, no. 1, pp. 85-94, 2008.

[25] H. L. Tidke, C. T. Aage, and J. N. Salunke, "Existence and uniqueness of continuous solution of mixed type integral equations in cone metric space," Kathmandu University Journal of Science, Engineering and Technology, vol. 7, no. 1, pp. 48-55, 1970.

[26] E. Beckenstein and L. Narici, "Subadditive separating maps," Acta Mathematica Hungarica, vol. 88, no. 1-2, pp. 147-167, 2000.

[27] M. A. Naimark, Normed Rings, Nordhoff, Groningen, Netherlands, 1959.

[28] V. Valvo, "On separating subadditive maps," Turkish Journal of Mathematics, vol. 39, pp. 168-173, 2015. 


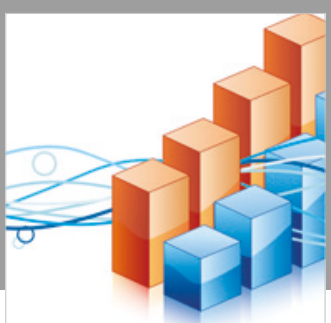

Advances in

Operations Research

\section{-n-m}
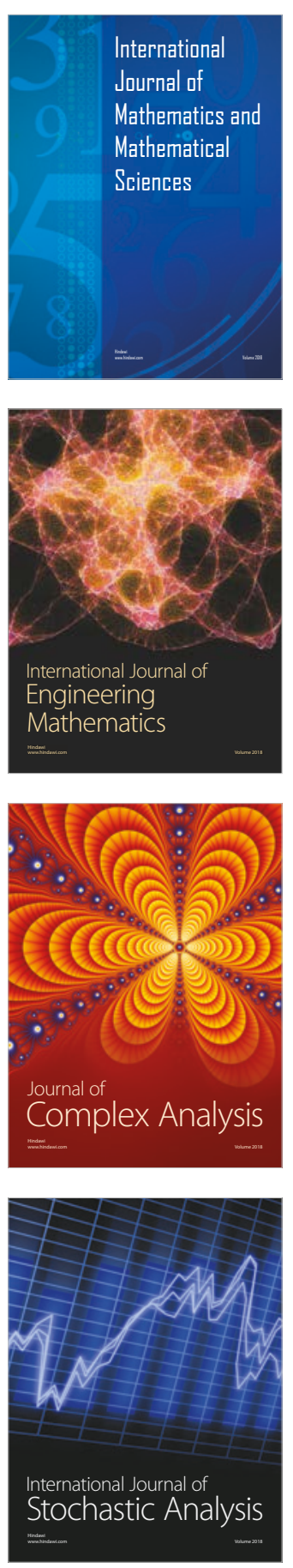
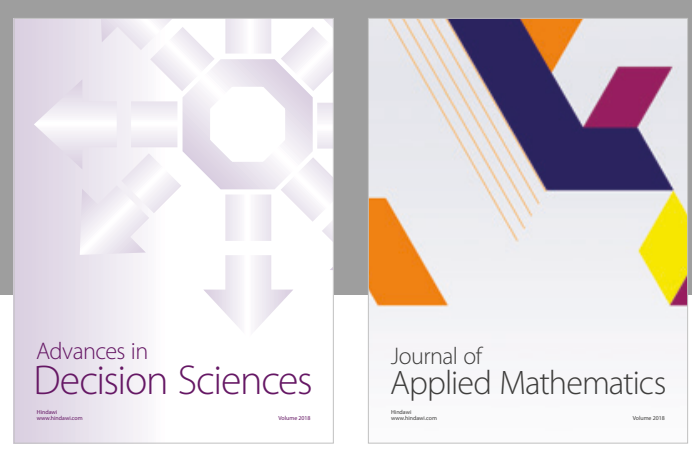

Journal of

Applied Mathematics
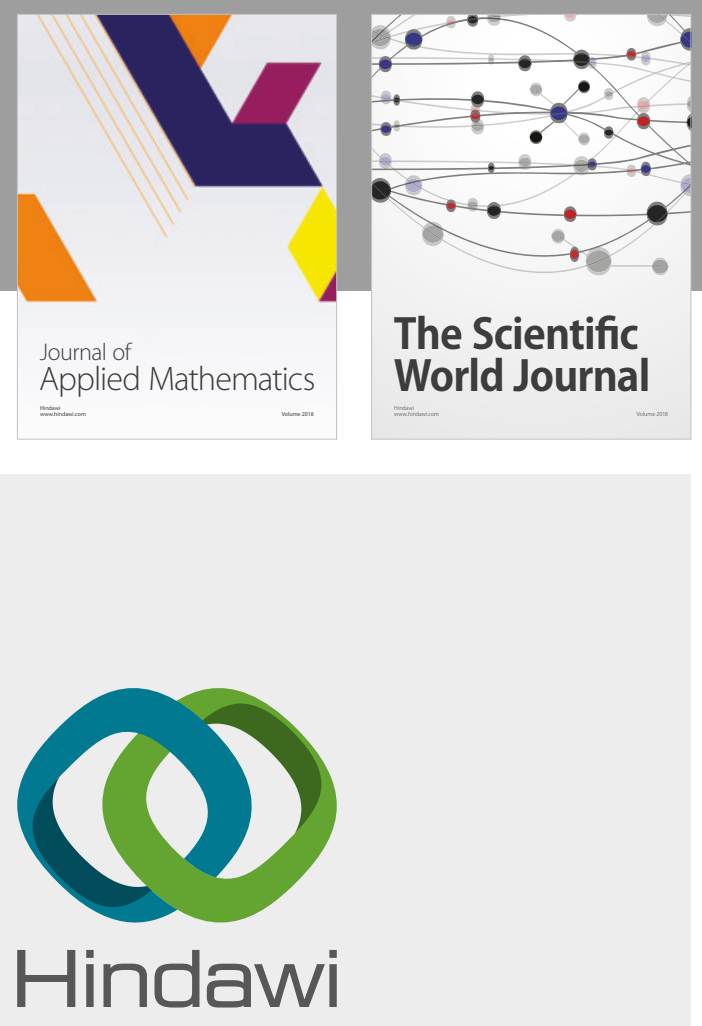

Submit your manuscripts at

www.hindawi.com

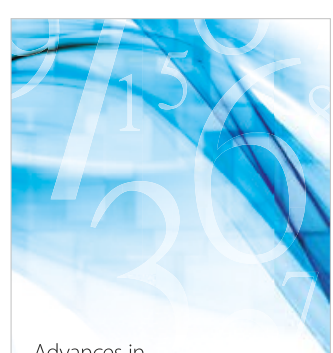

Advances in
Numerical Analysis
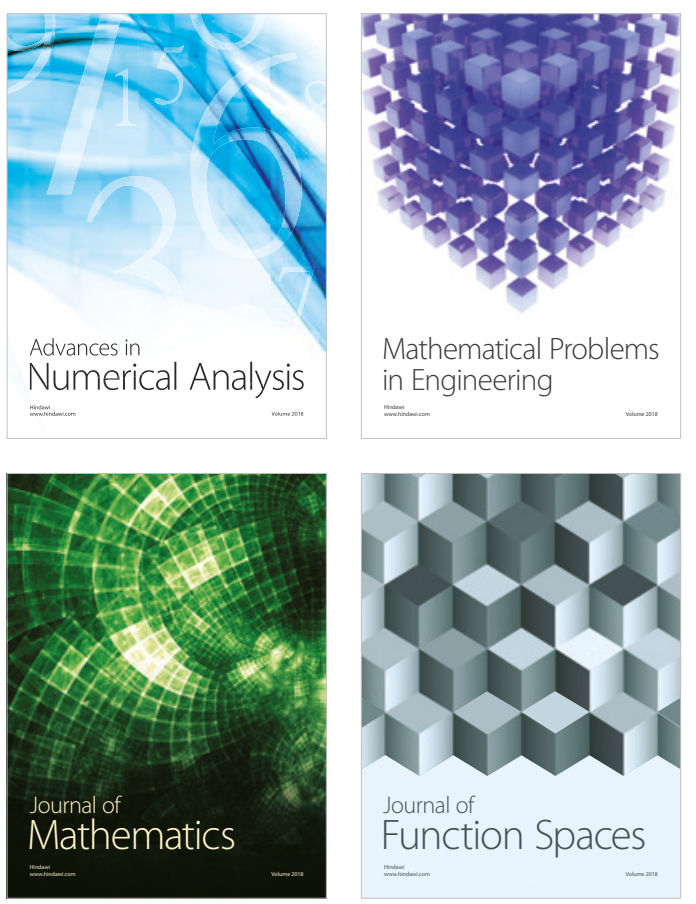

Mathematical Problems in Engineering

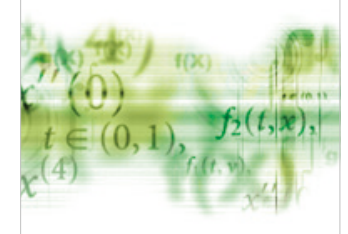

International Journal of

Differential Equations

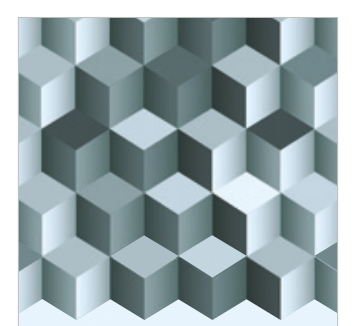

Journal of

Function Spaces

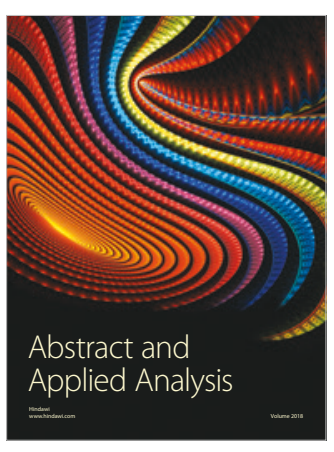

The Scientific

World Journal

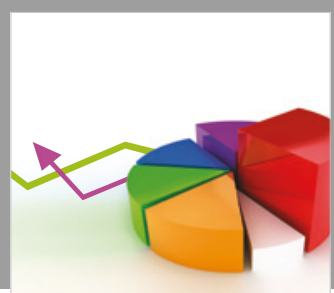

Journal of

Probability and Statistics
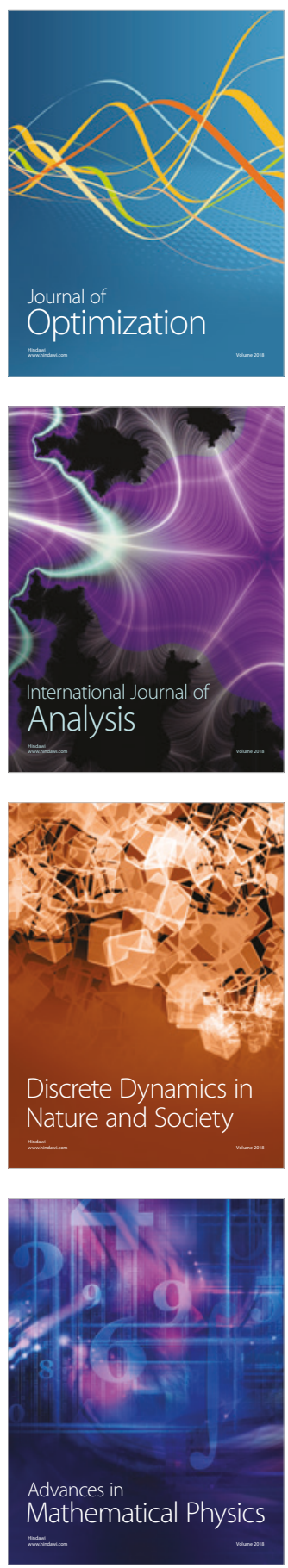